\title{
Transplantation de neurones embryonnaires dans le cervelet de souris
}

\section{Restauration de l'intégrité cérébelleuse chez des souris avec ataxie hérédo-dégénérative}

La mise en place de connexions synaptiques complexes et spécifiques entre des neurones embryonnaires greffés et les neurones d'un hôte adulte est possible. L'implantation de tissu provenant du cervelet d'embryons dans le cervelet d'une souris adulte dépourvue génétiquement de cellules de Purkinje aboutit à la migration des cellules de Purkinje du greffon vers les sites dépeuplés. Une fois en place, les neurones greffés établissent un réseau de connexions avec les neurones de l'hôte. Des signaux chimiotactiques en rapport avec l'absence de cellules de Purkinje seraient responsables de la migration cellulaire, du rétablissement du réseau synaptique et de la croissance guidée des axones vers leurs cibles. Les cellules transplantées semblent suivre normalement leur programme de différenciation et imposer aux cellules adultes de l'hôte une plasticité nécessaire à la constitution des circuits neuronaux.

\section{Constantino Sotelo}

\section{ADRESSE}

C. Sotelo: Docteur en médecine. Directeur de recherche au Cnrs. Directeur de l'U.106 de l'Inserm. laboratoire de neuromorphologie, Inserm L'. 106, Hôpital de la Salpétrière, 47, bd de l'Hôpital, 75013 Paris, France. 'impossibilité pour les neurones de proliférer empêche leur renouvellement. Les recherches effectuées au cours des dix dernières années sur la neur()transplantation expérimentale laissent entrevoir la possibilité d'un remplacement des neurones manquants dans le cerveau adulte, par implantation de neurones pris chez des embryons isogéniques, juste après la fin de leur dernière mitose, phase de leur développement qui permet leur dissociation et leur implantation sans entraîner leur mort. Dans certains cas bien précis [ 1, 2] ces implantations neuronales ont permis une récupération fonctionnelle partielle. Ces résultats, bien que limités, la voie à une possible chirurgie réparatrice du système nerveux dans les cas de maladies neurodégénératives, tout particulièrement dans la maladie de Parkinson.

Le propos de cet article n'est pas d'enoffrent un espoir nouveau, ouvrant visager l'aspect clinique des neurotransplantations, ou de discuter le bien-fondé de leur application thérapeutique, mais de les considérer comme un outil expérimental permettant d'étudier le rôle des interactions cellulaires dans l'ontogenèse et dans la plasticité nerveuse. Nous analyserons ici les mécanismes cellulaires impliqués dans la substitution neuronale, en particulier dans les cas de transplantations cérébelleuses qui sont réalisées dans notre laboratoire avec R.M. Alvarado-Mallart.

\section{Organisation cérébrale : l'électrique et le chimique}

De manière un peu simpliste, on peut distinguer dans le système nerveux central deux types d'organisation qui se chevauchent.

- Les réseaux neuronaux et la transmission synaptique. Depuis la formulation de Cajal [3] de la théorie neuronale, le système nerveux est 


\section{RÉFÉRENCES}

1. Björklund A, Segal M, Stenevi U. Functional reinnervation of rat hippocampus by locus coeruleus implants. Brain Res 1979; $170: 409-26$.

2. Dunnett SB, Björklund A, Stenevi U, Iversen SD. CNS transplantation : structural and functional recovery from brain damage. Prog Brain Res $1982 ; 55$ : 431-44.

3. Ramón y Cajal S. La fine structure des centres nerveux. Proc R Soc Lond (Biol) 1894 ; 55 : 444-68.

4. Katz B. Nerve, muscle and synapse. New York: McGraw Hill, 1966.

5. Barchas JD, AkilH, ElliolGR, Holman RB, Watson SJ. Behavioral neurochemistry: neuroregulators and behavioral states. Science 1978; 200 : 964-73.

6. Mobley P, Greengard P. Evidence for widespread effect of noradrenaline on axon terminals in the rat frontal cortex. Proc Natl Acad Sci USA 1985 ; 82 : 945-7.

7. Kesslak JP, Nieto-Sampedro M, Globus J, Cotman CW. Transplants of purified astrocytes promote behavioral recovery of frontal cortex ablation. Exp Neurol 1986 ; 92 : 377-90.

8. Björklund $\mathrm{A}$, Lindvall $\mathrm{O}$, Isacson $\mathrm{O}$, et al. Mechanisms of action of intracerebral neural implants: studies on nigral and striatal grafts to the lesioned striatum. TINS 1987; $10: 509$ 16

9. Madrazo I, Drucker-Colin R, Diaz V, Martinez-Mata J, Torres C, Becerril JJ. Open microsurgical autograft of adrenal medulla to the right caudate nucleus in two patients with intractable Parkinson's disease. $N$ Engl J Med 1987; 316: 831-4.

10. Mullen RJ, Eicher EM, Sidman RL. Purkinje cell degeneration, a new neurological mutation in the mouse. Proc Natl Acad Sci

conçu comme un très vaste ensemble de neurones constitués en réseaux neuronaux, d'une complexité quasiinextricable, mais d'une extrême précision. Les informations nerveuses transitent à l'intérieur de ces réseaux sous forme de messages électriques, qui sont transférés d'un neurone à l'autre par l'intermédiaire de structures spécialisées, les synapses. A leur niveau, l'arrivée de l'influx nerveux induit la libération d'une substance chimique, le neurotransmetteur, qui en diffusant dans la fente synaptique va contacter le récepteur post-synaptique, produisant la réponse électrique du neurone post-synaptique. Les caractéristiques essentielles de ce type de communication sont: sa rapidité (de l'ordre de la milliseconde) [4] et sa spécificité ponctuelle. L'activité de chaque neurone est le résultat de l'organisation spatio-temporelle des activités synaptiques dont il est le siège. En d'autres termes, la fonction du système est déterminée par l'organisation des afférences et des efférences à chaque neurone. Ces caractéristiques sont donc propres aux systèmes dits de «projection point-par-point » comme la majorité des systèmes sensoriels et moteurs, dans lesquels chaque neurone n'entre en contact synaptique qu'avec un nombre relativement réduit de cibles.

- Systèmes régulateurs et action paracrine de certaines classes de neurones. A côté des neurones intégrés dans des réseaux et communiquant par des synapses conventionnelles, il y a d'autres systèmes plus diffus qui ne transportent pas de messages spécifiques et qui interviennent comme régulateurs ou modulateurs de l'activité synaptique [5]. Ces systèmes sont caractérisés par le fait qu'un nombre réduit de neurones, localisés dans des noyaux précis, envoient leurs projections sur de très vastes territoires terminaux. Ainsi, un seul neurone d'un de ces «systèmes à projection diffuse» va pouvoir en influencer des centaines de milliers d'autres selon une topographie stricte, mais dans laquelle la spécificité synaptique n'est pas indispensable, car ils peuvent agir de manière paracrine $^{*}[6]$. L'exemple le plus

\footnotetext{
* Paracrine: action d'une substance sécrétée sur une cellule proche, par diffusion dans le milieu extracellulaire.
}

représentatif de «système à projection diffuse » est l'ensemble des systèmes monoaminergiques. L'un d'entre eux est le système dopaminergique, dans lequel la dopamine peut, outre sa fonction synaptique, diffuser dans l'espace extracellulaire sur de longues distances et affecter, selon le «principe de l'arrosage», tous les neurones équipés de récepteurs adéquats.

\section{Neurotransplantations : le cervelet comme modèle}

La majorité des travaux expérimentaux réalisés à ce jour ont porté sur des implantations substitutives de neurones appartenant aux «systèmes diffus », où de fait, il est concevable d'obtenir des restaurations fonctionnelles partielles, sans qu'il soit nécessaire pour autant d'obtenir la reconnexion du circuit lésé. Dans ces systèmes globaux, l'amélioration fonctionnelle peut en effet résulter de mécanismes généraux (libération des substances trophiques par des cellules du cerveau embryonnaire [7]) et surtout de mécanismes spécifiques (libération tonique de la substance neuro-active manquante par les neurones implantés même en l'absence d'intégration synaptique [8]).

Les expériences réalisées par Björklund et al. [8] sur un modèle simple de maladie de Parkinson chez le rat, vont tout à fait dans cette direction. Des rats ayant reçu une injection nigrale unilatérale de 6-OHDA, qui détruit tous les neurones à dopamine (DA), ont été greffés avec des neurones mésencéphaliques fotaux dans le néostriatum (territoire terminal de la voie DAergique nigrostriatale). Un faible pourcentage des neurones implantés développe un phénotype DAergique et, malgré l'absence de régulation rétroactive adéquate, sont capables par libération tonique de la DA d'améliorer certains comportements déficitaires, en particulier le comportement moteur spontané et sensorimoteur. Ces importants travaux ont été à l'origine de l'application clinique de la neurotransplantation aux patients parkinsoniens [9].

Le cervelet de la souris porteuse de la mutation Purkinje cell degeneration (pcd) [10] offre un modèle optimal pour déterminer si l'on peut 


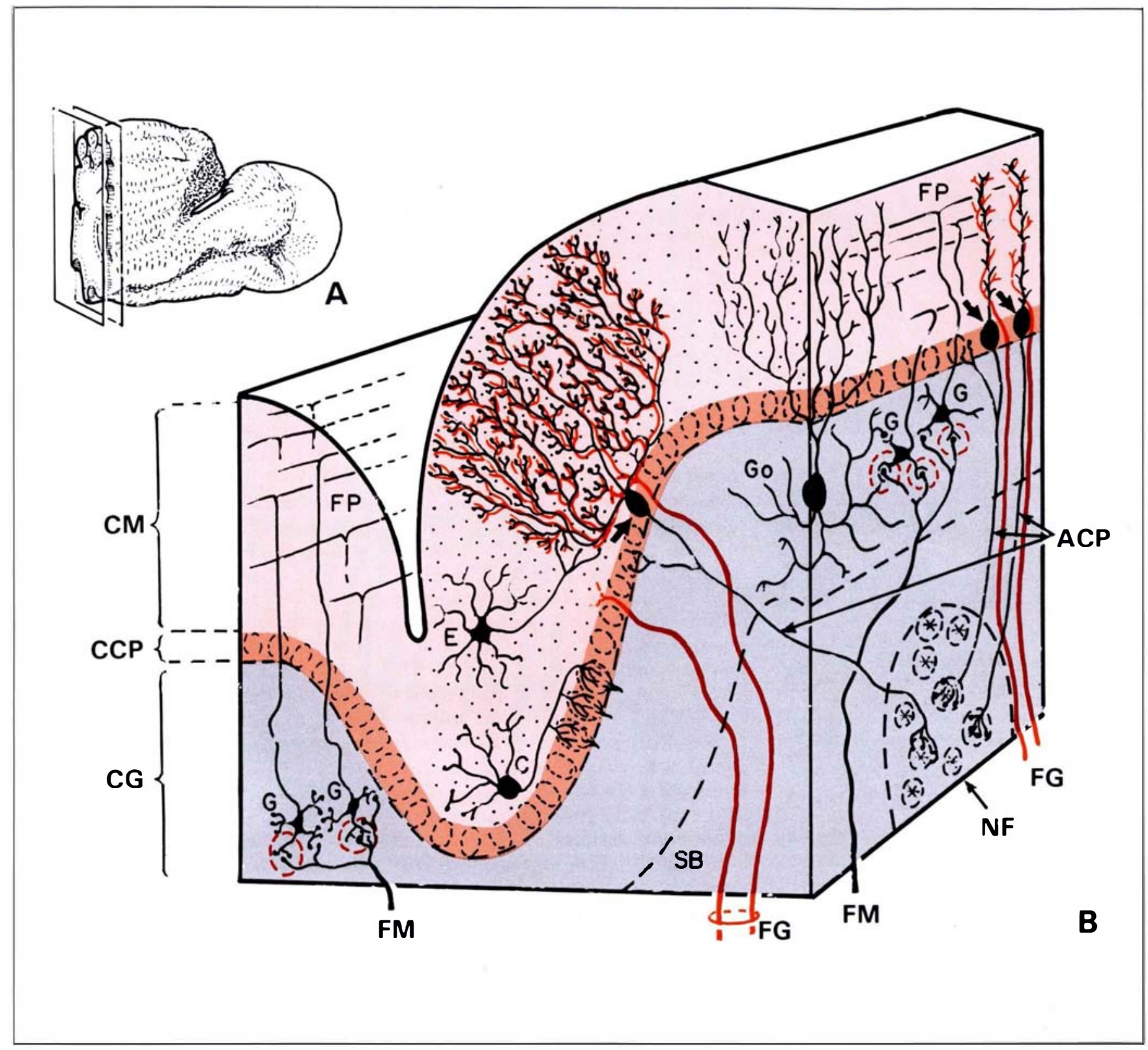

Figure 1. Représentation schématique du cervelet et des circuits cérébelleux. A. Représentation tridimensionnelle d'un hémicervelet de souris, montrant la foliation corticale. Les deux plans sagittaux délimitent la coupe où le folium illustré en $B$ a été pris. B. Le cortex du cervelet est une structure laminaire composée de trois couches : couche moléculaire (CM), couche des cellules de Purkinje (CCP) et couche granulaire (CG). Le centre du folium est occupé par la substance blanche $(S B)$, et dans sa profondeur se trouvent les noyaux cérébelleux; dans cette coupe vermienrie le noyau médial ou fastigial (NF) est illustré. Seulement cinq types de neurones (cellules de Purkinje - flèches -, cellules des grains - G - cellules étoilées - E cellules en corbeille - C - et cellules de Golgi - Go -) sont disposés dans le cortex cérébelleux de manière rigoureusement géométrique. Deux afférences excitatrices: fibres grimpantes (FG) (en rouge) et fibres moussues (FM) apportent à ce cortex une information venue des organes des sens ou du cortex cérébral; les ordres donnés par le cortex cérébelleux sortent par une seule efférence constituée par les axones des cellules de Purkinje (ACP), se projetant sur les neurones des noyaux profonds (astériques). Les fibres grimpantes entrent en contact synaptique avec les cellules de Purkinje dans une relation numérique constante: une fibre grimpante - une cellule de Purkinje. Les fibres moussues sont reliées aux cellules de Purkinje par l'intermédiaire de cellules relais, qui sont les grains. Fibres moussues et dendrites des grains forment les glomérules cérébelleux (encerclés pointillés rouges). Les grains, par leurs axones - les fibres parallèles (FP) - atteignent les cellules de Purkinje. Les interneurones inhibiteurs modulent l'activité du circuit - les cellules étoilées et en corbeille par des synapses directes avec les cellules de Purkinje, et les cellules de Golgi par des synapses avec les dendrites des grains dans les glomérules. 


\section{RÉFÉRENCES}

11. Sotelo C. Alvarado-Mallart RM. Growih and differentiation of cerebellar suspensions tuansplanted into the adult cerebellum of mice with heredodegenerative alaxia. Proc vall Acad Sci L'SA 1986:83: 1135-9.

12. Sotelo C. Aluarado-Mallart R.M. Reconsuruction of the defective cerebellar circuitry in adult Purkinje cell degeneration mutant mice by Purkinje cell replacement through transplantation of solid embryonic implants. Neuroscience $1987: 20: 1$-22.

13. Holmes 6 . A form of familial degeneration of the crebellum. Brain 1907: 30: 46689

14. Sotelo C: Aluarado-Mallart RM. Embryonic and adulı neurons interact 10 allow Purkinje cell replacement in mutant cerebellum. Nature 1987: 327 : 421-3.

15. Ramón y Cajal S. Algunas observaciones favorables a la hipótesis neurotrópica. Trab Inst Cajal Invest Biol 1910:8: 63-135.

16. Rakic P. Neuronal-glial interaction during brain development. TIN'S 1981; 4: 184-7.

17. Sotelo C. Aluarado-Mallart R.M. Cerebellar transplantations in adult mice with heredo-degenerative ataxia. Ann $\mathrm{N}^{\prime}$. Acad Sci $1987: 195: 242-66$

18. Sotelo C. Alvarado-Mallart RM. Integration of grafted Purkinje cells into the host cerebellar circuitry in pcd mutant mouse. Prog Brain Res 1988: 7f (sous presse).

19. Sotelo C. Purkinje cell ontogens: formation and maintenance of spines. Prog Brain Res 1978: $48: 149-70$.

20. Gardetce R, Alvarado-Mallart R.M, Crepel F. Sotelo C. Electrophỵsiological demonstration of a synaptic integration of transplan. ted Purkinje cells into the cerebellum of the adult Purkinje cell degeneration mutant mouse. Neuroscience 1988; 24: 777-89.

21. Harris WA. Homing behaviour of axons in the embryonic vertebrate brain. Nature $1986: 320: 266-9$. reconstituer un circuit neuronal cablé «point par point» (figure 1) par des implants, contenant des cellules cérébelleuses prélevées sur des embryons isogéniques de 12 jours $[11,12]$. Chez le mutant $p c d$, après un développement normal du cervelet, les cellules de Purkinje - élément pivot du cortex cérébelleux à l'origine des efférences cortico-nucléaires - dégénèrent entre la $3^{\mathrm{e}}$ et $6^{\mathrm{e}}$ semaine après la naissance. Cette atrophie cérébelleuse ressemble donc aux atrophies cortico-cérébel 'euses (ou centrifuges) familiales humaines [13].

Dans un tel système, la récupérat ion fonctionnelle repose sur la possibilité d'obtenir du greffon une substitution des cellules de Purkinje, qui à leur tour réussissent leur intégration synaptique complète dans le cervelet de l'hôte, permettant la reconnexion cortico-nucléaire (figure 1). En un sens, il est demandé aux cellules de Purkinje implantées de poursuivre leurs étapes de développement, et aux neurones adultes de l'hôte de devenir des partenaires appropriés pour fournir les interactions cellulaires requises à la formation du circuit cérébelleux.

L'étude du cervelet des mutants pcd implantés à l'âge d'au moins deux mois, entre un jour et quatre mois après transplantation, nous a permis de suivre le cheminement des cellules de Purkinje implantées, d'établir la chronologie de leur développement, de constater que leur intégration synaptique pouvait être complète mais avec des limites, et d'analyser les mécanismes cellulaires qui soustendent cette intégration [14]

Migration sélective des cellules de Purkinje greffées. L'implant contient des neurones embryonnaires et des précurseurs de toutes les catégories cellulaires qui composent le cervelet. Cependant, les cellules de Purkinje sont les seules nécessaires à la restauration du circuit pcd. I'ne des découvertes majeures de cette étude a été d'établir que le neurotropisme[15] du cervelet déficitaire exerce une attraction spécifique pour les cellules de Purkinje, les seules qui quittent l'implant à son interface avec l'hôte et qui envahissent la couche moléculaire de ce dernier. Les autres populations cellulaires restent confinées dans un greffon résiduel de localisation variable (figure 2). La migration des cellules de Purkinje immatures est limitée, ne courrant qu'environ $700 \mu \mathrm{m}$ (micromètres) de chaque côté de l'implant. La nature, chimique ou électrique, de l'effet neurotropique exercé par la couche moléculaire du cervelet $p c d$ n'est pas connue. Ce neurotropisme est lié à l'absence en cellules de Purkinje, car il est nul quand ces neurones sont présents, comme c'est le cas chez la souris normale.

L'ne étude suivie dans le temps [14] a permis de déceler les routes migratoires des cellules de Purkinje. Quatre jours après l'opération, l'implant est en continuité avec le cervelet receveur, et un courant migratoire se forme à la surface des folia ${ }^{* *}$, entre la membrane basale sous-piale et la membrane limitante gliale. Après six à sept jours, le courant migratoire tangentiel a atteint son extension maximale $(700 \mu \mathrm{m})$ et les cellules de Purkinje qui le composent changent de polarité, envahissant radialement en suivant les palissades gliales des fibres de Bergmann*** - la couche moléculaire de l'hôte. Ainsi, malgré la direction aberrante de la migration, les mécanismes de reconnaissance entre neurones et axes gliaux [16], qui sont essentiels pendant l'ontogenèse, restent opérationnels chez l'adulte implanté et permettent l'arrivée des neurones manquants à leur destination. L'arrêt de la migration six à sept jours après transplantation, malgré la présence des cellules de Purkinje dans les greffons résiduels, impose une limitation à l'étendue de la restauration. Dans nos meilleurs cas, seulement $15 \%$ du volume de la couche moléculaire $\mathrm{d}$. cervelet pcd ont pu être pourvus en cellules de Purkinje greffées [17].

Différenciation dendritique des cellules de Purkinje greffées. Au cours de leur migration radiale, ces cellules sont bipolaires, et quand leur prolongement actif arrive à l'interface entre couches moléculaire et granulaire, le processus migratoire s'arrête. Les mécanismes qui gouvernent cet

\footnotetext{
* Folium: circumvolution de l'écorce cérébelleuse.

** Fibres de Bergmann: prolongements externes d'une catégorie de glie radiale (les cellules épithéliales de Golgi), spécifiques de
} la couche moléculaire du cenielet adulte. 


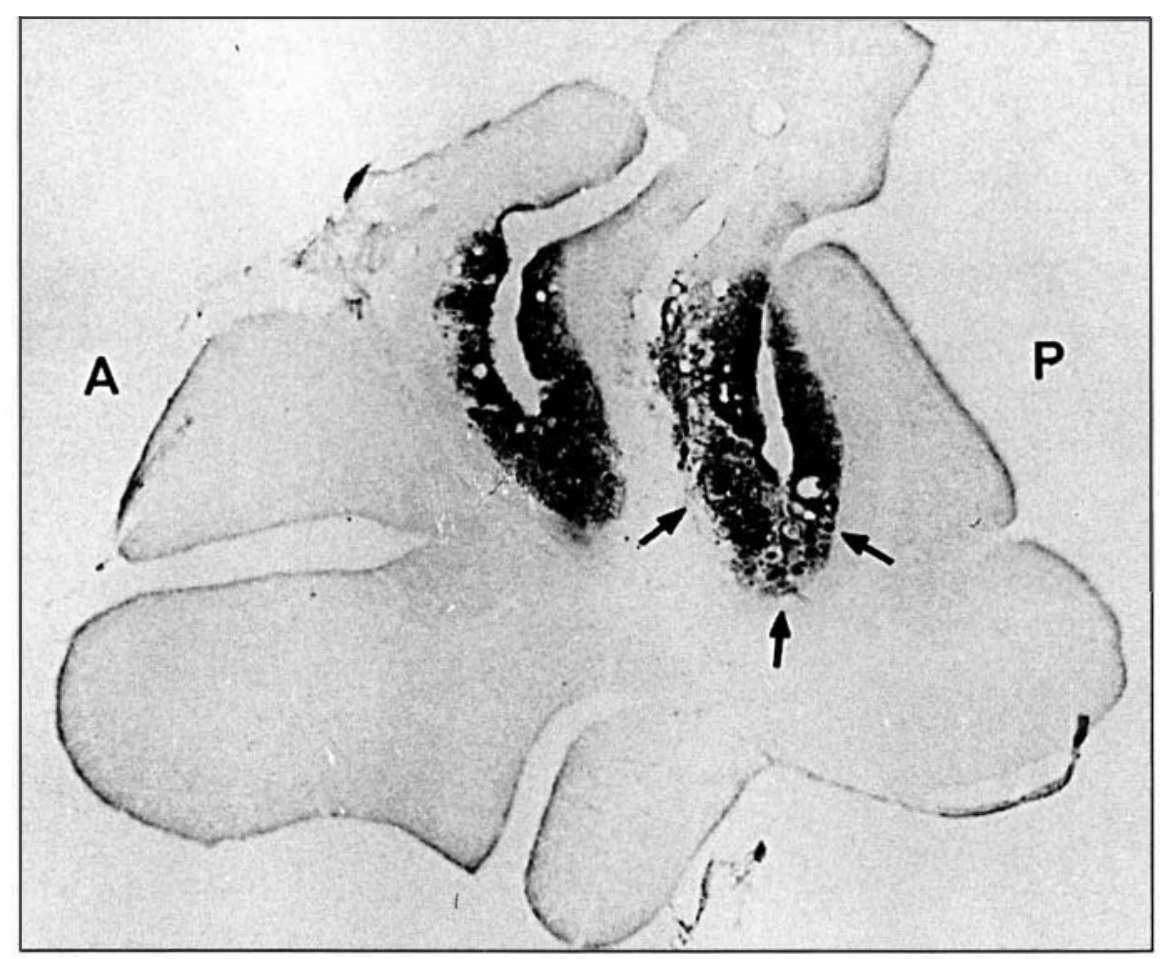

Figure 2. Coupe sagittale du cervelet de souris porteuse de la mutation pcd, deux mois après transplantation d'un greffon solide. Les cellules de Purkinje implantées sont visualisées par immunohistochimie, à l'aide d'un anticorps anti-calbindine. Les flèches délimitent la région contenant le greffon résiduel. Noter la localisation des cellules de Purkinje implantées qui ont réussi leur migration dans des régions bien déterminées de la couche moléculaire du cervelet hôte. A : antérieur ; $P$ : postérieur. $(X 50)$. situées au milieu de la couche moléculaire montrent un aspect étoilé [ 11 , 12]. Malgré ces altérations, les dendrites réussissent à acquérir les deux signes distinctifs qui les caractérisent normalement chez l'adulte: (1) elles sont confinées au plan sagittal, perpendiculaire aux faisceaux des fibres parallèles de l'hôte; (2) elles sont composées d'un compartiment proximal et d'un autre distal [19]. Cela laisse supposer que les interactions synaptiques qui ont lieu pendant cette dendritogenèse, entre d'une part les fibres parallèles et les fibres grimpantes adultes de l'hôte, et d'autre part les dendrites en croissance des neurones implantés suivent les mêmes règles que celles opérationnelles au cours de l'ontogenèse cérébelleuse.

L'étude suivie dans le temps confirme cette proposition. Quinze jours après transplantation, les dendrites des cellules de Purkinje sont déjà confinées dans le plan sagittal, et leurs segments distaux sont hérissés d'épines (figure 3), comme c'est le cas d'une cellule de Purkinje d'une souris âgée de sept jours.

Intégration synaptique des cellules de Purkinje greffées dans le circuit du cortex cérébelleux et leur régula- arrêt doivent être en relation avec le fait que la couche granulaire n'est, normalement, le siège ni des corps cellulaires ni des dendrites des cellules de Purkinje. Le cône de croissance dendritique ne reconnaît pas la couche granulaire comme environnement «permissif » et à proximité de celle-ci se déclenchent les informations qui vont bloquer la phase migratoire des neurones [18]. La conséquence de ce bloquage est que les corps cellulaires des cellules de Purkinje greffées restent en position ectopique dans les $4 / 5^{\text {es }}$ superficiels de la couche moléculaire. C'est à partir de ce moment que les cellules de Purkinje greffées, comme au cours de leur ontogenèse normale, vont fabriquer leurs arbres dendritiques. Les altérations observées dans les arbres dendritiques des cellules de Purkinje implantées s'expliquent par l'ectopie de leurs corps cellulaires. Celles placées sous la surface du folium ont souvent un arbre dendritique inversé. En revanche, celles $\mathrm{m} / \mathrm{s} n^{\circ} 8$ vol. 4 , octobre 88

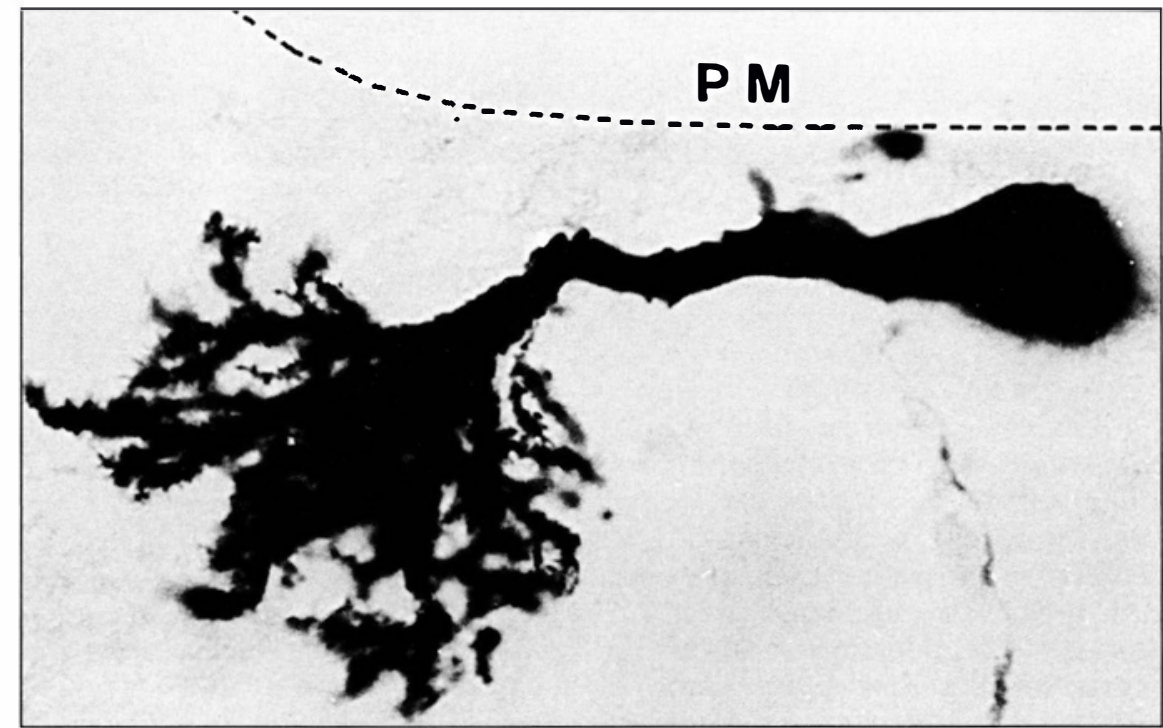

Figure 3. Coupe sagittale du cervelet de souris pcd, 15 jours après la greffe neuronale. L'âge réel des cellules de Purkinje greffées est donc de sept jours après la naissance. Cette micrographie illustre une de ces cellules de Purkinje à la limite du front de migration. II faut remarquer que malgré sa position ectopique sous la pie-mère (PM) et sa disposition horizontale, son arbre dendritique est très semblable à celui d'une cellule de Purkinje d'une souris normale âgée de sept jours. (X 990). 
tion par des afférences spécifiques. L'étude morphologique [11, 12] et élecurophysiologique [20] des cellules de Purkinje greffées qui ont atteint la couche moléculaire du cervelet $p c d$ montre que les afférences propres à ces neurones réussissent à établir des synapses qui gardent leur spécificité et leur fonction. Chaque cellule de Purkinje reçoit, comme dans le cervelet normal, des afférences des fibres grimpantes sur le compartiment dendritique proximal (figure 4). En outre, la relation numérique constante chez l'adulte: une fibre grimpante - une cellule de Purkinje, est maintenue (figure 6). Les fibres moussues, à travers leurs cellules relais que sont les grains et leurs axones, les fibres parallèles, peuvent transmettre leurs informations aux cellules de Purkinje greffées, selon la modalité propre au cervelet normal (figures 4 et 6 ). Enfin, les interneurones inhibiteurs, cellules étoilées et en corbeille, malgré l'absence des «pinceaux » autour du segment initial de l'axone des cellules de Purkinje greffées réussissent à former des synapses axo-axoniques, axo-dendritiques et axo-somatiques (figure 5) avec les neurones implantés, comme dans le cervelet normal. Ces synapses induisent des réponses inhibitrices, transitant par la voie trisynaptique: fibres moussues - fibres parallèles - axones des cellules en corbeille et étoilées (figure 6). Ces observations montrent que le circuit du cortex cérébelleux est restauré, suite au remplacement des neurones manquants par des neurones homologues greffés, avec intégration synaptique complète.

La mise en place des contacts synaptiques entre les axones des neurones adultes et les cellules de Purkinje implantées va se faire selon une chronologie et un schéma de développement propre à ces dernières [14]. Il semble évident que c'est la présence des neurones embryonnaires qui impose aux neurones adultes les règles de leur intégration dans le circuit déficitaire, comme s'ils étaient contrôlés par une horloge interne, indépendante des signaux d'environnement. Cette hypothèse offre une explication aux limites de l'étendue de la migration: dans leur programme intrinsèque, les cellules de Purkinje peuvent migrer pendant un

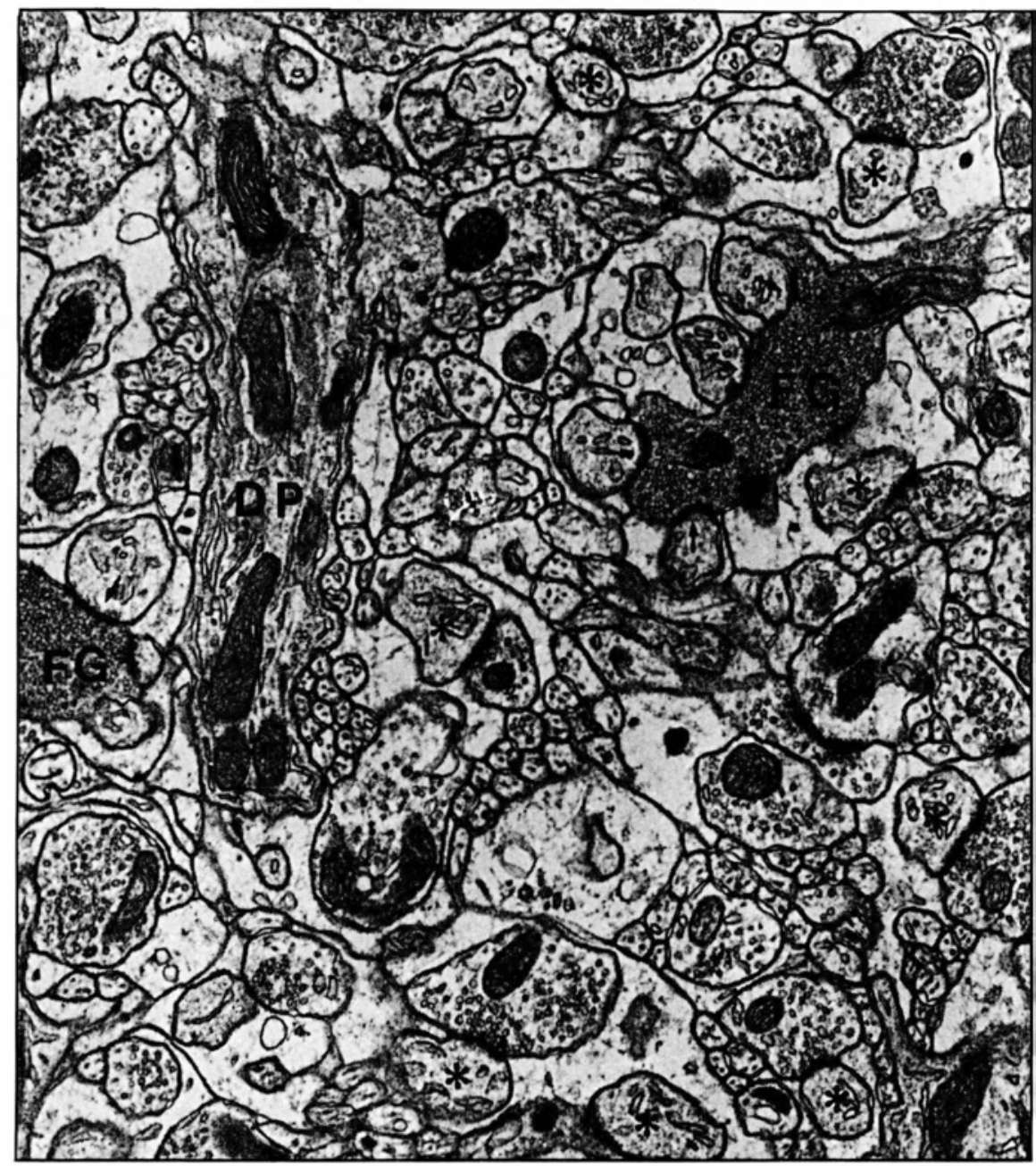

Figure 4. Micrographie électronique de la couche moléculaire du cervelet d'une souris pcd, deux mois après transplantation. Cette micrographie illustre les afférences synaptiques que reçoivent les dendrites des cellules de Purkinje greffées. Une branche dendritique secondaire (DP) émet des épines qui sont contactées (flèches) par des varicosités appartenant à des fibres grimpantes (FG). En outre, les épines distales (astérisques) sont post-synaptiques à des fibres parallèles. $(\times 19000)$.

Figure 6. Enregistrements intracellulaires in vitro des cellules de Purkinje greffées obtenus sur des tranches cérébelleuses, un mois après transplantation, après stimulation électrique de la substance blanche à la base du folium. A. Réponse antidromique typique (quatre balayages superposés). B. Activité synaptique entre fibre grimpante de l'hôte et cellule de Purkinje greffée enregistrée dans les cellules de Purkinje empalées. Le registre montre une réponse tout-ou-rien, typique des synapses entre fibre grimpante et cellule de Purkinje dans le cervelet normal (complex spike) (cinq balayages superposés). Son caractère toutou-rien montre que les neurones greffés sont innervés, comme les normaux, par une seule fibre grimpante. C. Exemple de réponse du système fibre moussue-fibre parallèle-cellule de Purkinje (flèche); une augmentation de l'intensité du stimulus provoque une réponse de fibre grimpante (tête de flèche). D. Potentiel postsynaptique inhibiteur moyen obtenu à partir de 20 enregistrements (voir [20]). 


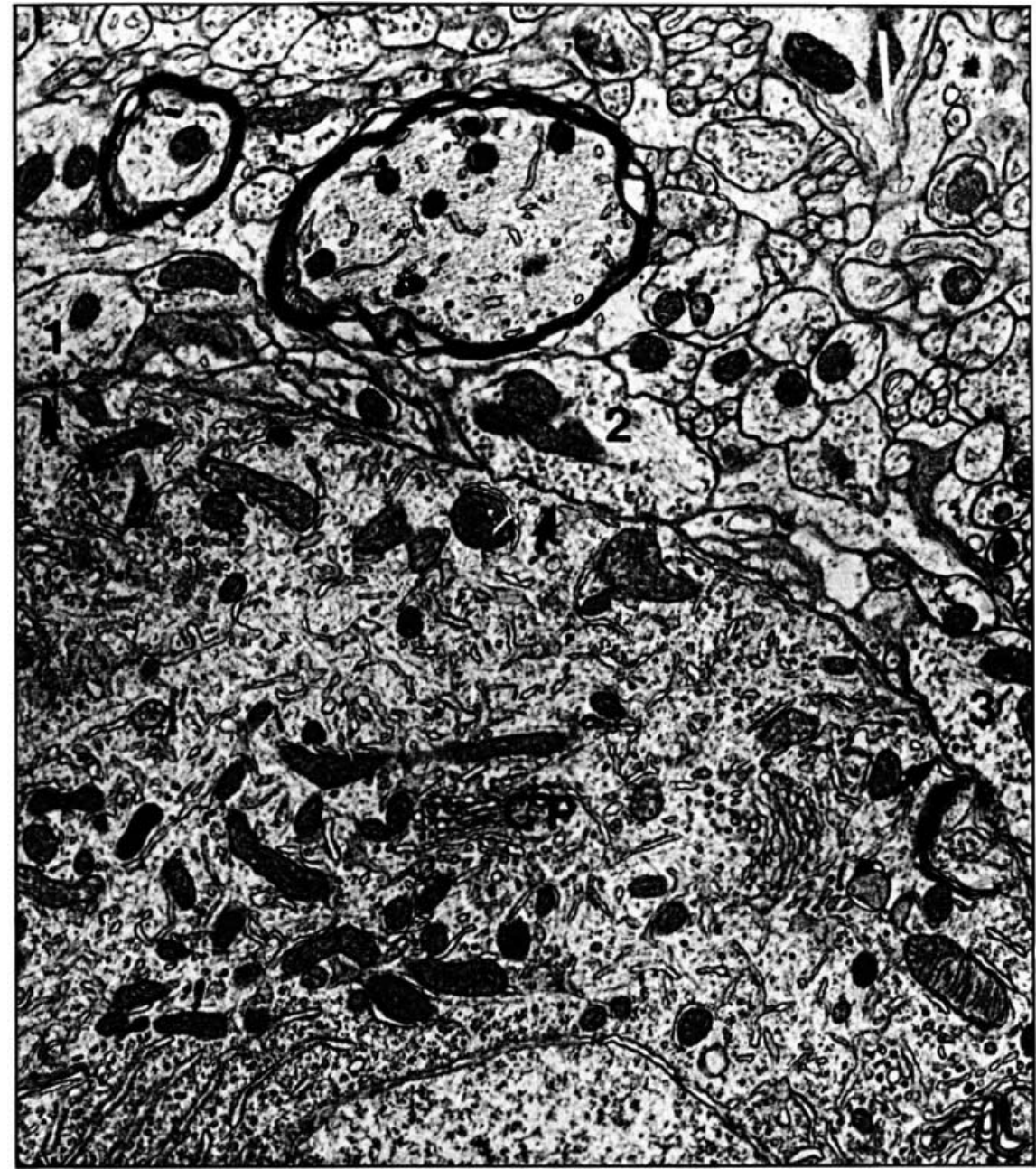

Figure 5. Cette micrographie électronique, prise dans le même matériel que sur la figure 4, montre le corps d'une cellule de Purkinje greffée (CP) en position ectopique au centre de la couche moléculaire. Ce corps cellulaire reçoit trois boutons (1-3), provenant des axones des cellules étoilées et en corbeille, qui établissent des synapses (flèches) avec le neurone greffé. $(\times 14000)$.

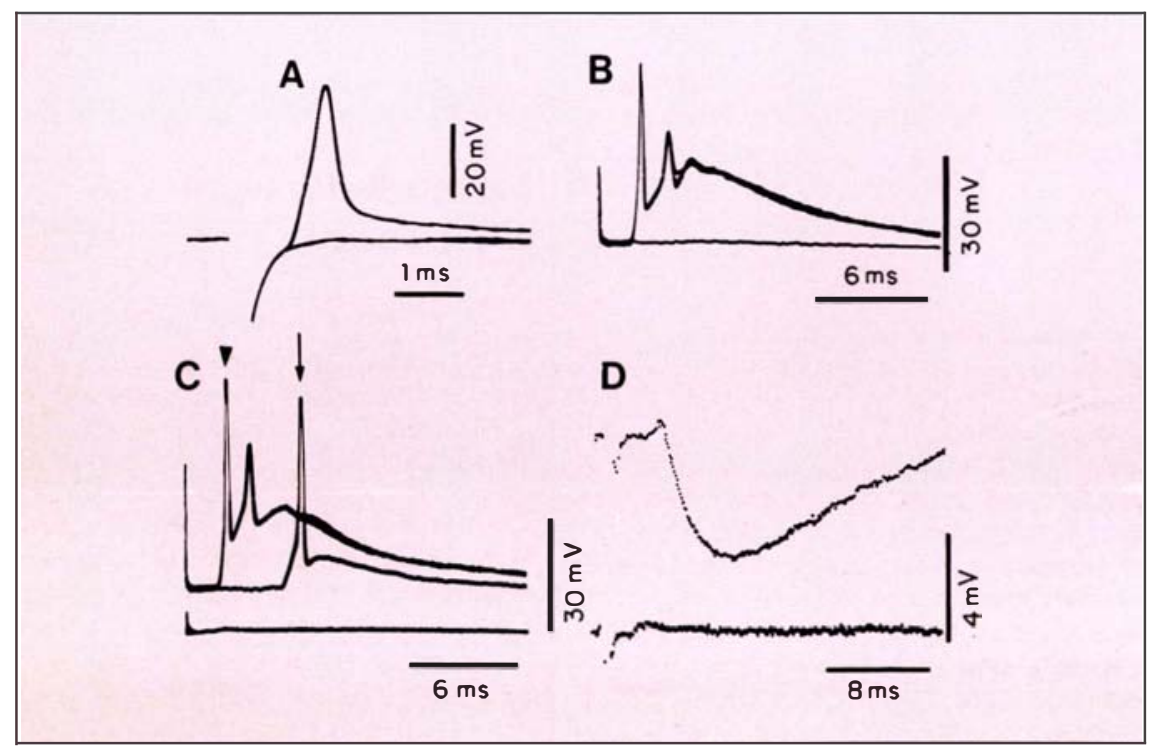

$\mathrm{m} / \mathrm{s} n^{\circ} 8$ vol. 4 , octobre 88 maximum de 6 jours après la fin de la prolifération, ce qui équivaut à l'âge réel de ces neurones 7 jours après transplantation, date à laquelle la migration a atteint son maximum. Reconnexion cortico-nucléaire : le problème de la croissance et de la navigation axonale. Pour aboutir à un certain degré de restauration fonctionnelle, il est nécessaire que les informations élaborées par le cortex cérébelleux puissent être transmises aux neurones cibles, localisés dans les noyaux cérébelleux profonds. En d'autres termes, il faut que les cellules de Purkinje implantées rétablissent la projection cortico-nucléaire. Ce point reste encore le plus critique car il présuppose la possibilité d'une croissance guidée (navigation) [21] des axones des cellules de Purkinje implantées, tout comme au cours de l'ontogenèse. La différence est, cependant, essentielle : la distance à parcourir est incomparablement plus longue chez l'adulte que chez l'embryon, et les molécules de signalisation probablement différentes, car l'environnement offert aux cônes de croissance est celui du cervelet adulte.

Ces difficultés expliquent que la reconstitution de la projection cortico-nucléaire pose encore des problèmes, car si certains axones des neurones transplantés réussissent indubitablement à innerver les noyaux profonds, ceci n'est pas un fait constant. La croissance et la navigation axonales sont contrôlées par des interactions spécifiques avec le tissu environnant, particulièrement par le neurotropisme positif exercé par les neurones cibles. Ici aussi, la nature du neurotropisme reste inconnue, et l'on peut invoquer la création d'un gradient chimique à partir des neurones des noyaux profonds qui ont perdu une partie importante de leur innervation et qui sécrèteraient des facteurs trophiques spécifiques aux cellules de Purkinje. Cette hypothèse expliquerait pourquoi les deux facteurs limitants de la reconnexion cortico-nucléaire sont: (1) la présence de neurones des noyaux profonds embryonnaires dans les greffons résiduels : car ils réussissent à attirer un bon nombre d'axones des cellules de Purkinje intégrées dans le cervelet hôte, les déviant de leur cheminement vers les noyaux profonds 
de cervelet $p c d$; (2) la distance entre la position du greffon et les noyaux profonds de l'hôte : quand cette distance est faible (environ 100 à 200 $\mu \mathrm{m})$, la projection cortico-nucléaire est massive. Quand elle est supérieure à $600 \mu \mathrm{m}$, la projection ne se forme pas.

\section{Conclusion}

Les implantations des neurones cérébelleux embryonnaires dans le cervelet $p c d$ adulte peuvent aboutir à l'intégration synaptique des cellules de Purkinje dans le circuit cortical et à la reconstitution de la projection cortico-nucléaire. Ces résultats sont importants car ils permettent d'envisager avec optimisme l'avenir des neurotransplantations en clinique humaine. Cependant, toutes les conditions ne sont pas encore réunies pour recommander ce type d'intervention comme chirurgie réparatrice des circuits organisés selon la modalité « point-par-point». En effet, l'efficacité de ce traitement dépend de l'étendue de la restauration et, pour le moment, les limitations rencontrées dans la migration des cellules de Purkinje, et surtout dans la navigation de leurs axones vers leurs cibles de l'hôte, font que cette étendue reste encore trop faible.

Ces recherches ont eu aussi une incidence sur l'évaluation des capacités adaptatives des neurones adultes. Les résultats rapportés indiquent que les signaux nécessaires à la migration, à la différenciation neuronale et à la synaptogenèse, qui existent chez les embryons, sont aussi exprimés chez les adultes; au moins dans le cas où leurs cellules nerveuses sont mises en contact avec des neurones embryonnaires. Cela laisse supposer que la présence de neurones embryonnaires dans un système nerveux adulte pourrait influencer la régulation de l'expression génétique de leurs neurones et cellules gliales en engendrant un microenvironnement «permissif » transitoire qui permettrait à ces cellules de retrouver certaines facultés perdues après l'ontogenèse et de participer avec succès au processus de substitution neuronale. Il s'agirait d'une facette encore inconnue de la plasticité neuronale, consécutive à des interactions entre neurones immatures et adultes

\section{Summary}

The degree of host integration of grafted embryonic neurons to obtain behavioral recovery depends on the nature of the lesioned system. In «global » systems (diffusely projecting, regulatory pathways), the grafted neurons may exert their functional effects by a paracrine mechanism involving diffuse secretion of the missing neuro-active substance into the appropriate terminal domains. Conversely, effective transplantations in «point-to-point» systems (pathways with precise wiring, interconnected through classical synapses) requires integration of grafted neurons into the host defective circuitry, with reconnection of disconnected input - output relationships. The work done with cerebellar grafts in mutant ( $p c d$ ) mouse with heredo-degenerative ataxia provides a demonstration that this level of precise reconstruction can take place, although with important limitations. Both the detailed timetable and the specific cellular interactions followed by grafted Purkinje cells during their host cerebellar integration are remarkably similar to those taking place during normal development. It is suggested that embryonic Purkinje cells induce in adult neural cells a new type of plasticity, that of recreating a permissive microenvironment for the synaptic integration of the grafted neurons, leading to the restoration of the impaired cerebellar circuit.

\section{TIRÉS A PART}

C. Sotelo. 